www.mdpi.com/journal/applsci

Article

\title{
The Effect of Trifluoroethanol and Glycerol on the Thermal Properties of Collagen Using Optical Displacement-Enhanced Heterodyne Polarimeter
}

\author{
Chien-Ming Wu ${ }^{1}$, Horn-Haw Chen ${ }^{1,2}$, Kai-Han Tseng ${ }^{1}$ and Hung-Wei Chen ${ }^{3, *}$
}

1 Department of Biomedical Engineering and Environmental Sciences, National Tsing Hua University, Hsinchu 30013, Taiwan; E-Mails: cmwu@mx.nthu.edu.tw (C.-M.W.); hornhchen@gmail.com (H.-H.C.); khtseng@gmail.com (K.-H.T.)

2 Center for Measurement Standards, Industrial Technology Research Institute (ITRI), Hsinchu 30013, Taiwan

3 Department of Electronic Engineering, National United University, Miaoli 36063, Taiwan

* Author to whom correspondence should be addressed; E-Mail: hwchen@nuu.edu.tw; Tel.: +886-37-382-528; Fax: +886-37-382-498.

Academic Editor: Philippe Lambin

Received: 20 July 2015 / Accepted: 2 November 2015 / Published: 13 November 2015

\begin{abstract}
An angular displacement-enhanced heterodyne polarimeter has been employed to investigate the interplay between trifluoroethanol (TFE) and glycerol on the thermal denaturation of type I collagen. The concentration of the collagen solution was fixed at $0.341(\mathrm{mg} / \mathrm{mL})$, and was heated from $25{ }^{\circ} \mathrm{C}$ to $55^{\circ} \mathrm{C}$. TFE solutions with concentrations of $5 \%, 10 \%, 15 \%, 20 \%, 40 \%$ and $80 \%(v / v)$ were prepared and the phase change was recorded for the determination of thermal denaturation. It was observed that the thermal denaturation temperature $\left(T_{d}\right)$ is decreased with increasing TFE concentration due to the partial cleavage of the triple-helical structure. With TFE concentration higher than $20 \%(\mathrm{v} / \mathrm{v})$, the degree of optical rotation appears to be nearly the same, reflecting that the collagen triple helices have been completely destructed. Moreover, the addition of glycerol in inhibiting the thermal denaturation of collagen is investigated. It has been shown that glycerol can improve the thermal denaturation of both collagen and TFE-mixed collagen. Experimental results show that, in the presence of $2 \mathrm{M}$ glycerol, the $T_{\mathrm{d}}$ of collagen remained at around $41.9{ }^{\circ} \mathrm{C}$, meanwhile the $T_{\mathrm{d}}$ of $20 \%(v / v)$ TFE-mixed collagen is significantly restored to $32.8{ }^{\circ} \mathrm{C}$.
\end{abstract}


Keywords: collagen protection; heterodyne polarimeter; thermal denaturation; trifluoroethanol; glycerol

\section{Introduction}

Collagen is the major component of the human proteome and plays a key role in the structural connection of tissues, such as ligaments, tendons, ocular sclera, bone, and skin. Collagen has a unique structure in which the hierarchical structure is composed of the triple helices consisting of twisted polypeptide chains. The collagen-derived biomaterials have been widely developed in the applications of medical cosmetology and tissue repairing, and regeneration engineering. In the preparation of these biomaterials, trifluoroethanol (TFE) is used as a co-solvent. For instance, collagen-glycosaminoglycan scaffold [1,2] and collagen nano-fibers [3] used for tissue reconstruction can be prepared from aqueous TFE mixture. Shanmugam et al. [4] have summarized the effect of TFE induced in proteins, which can stimulate the conformational transition between random coil, $\alpha$-helix, and $\beta$-sheet in proteins and peptides. Shanmugam et al. [4] also investigated the effect of TFE on type I collagen structure using circular dichroism (CD) and Fourier transfer infrared (FTIR) spectroscopy techniques. The results of thermal melting revealed that the stability of triple helix in collagen was decreased even at a low concentration of TFE $(5 \%, v / v)$ by the observation of decreased optical density [4]. Furthermore, the results of the turbidity measurements revealed that the collagen structure is dissociated by inhibiting the fibril formation under the circumstance of higher TFE concentration [4].

Glycerol is a hyperosmotic agent used to adjust the optical scattering properties of turbid tissues. Yeh et al. [5] have reported that the use of glycerol screens intermolecular interactions between low-order structure in collagen and leads to fiber disassembly. D. Cooper et al. [6] used glycerol as a stabilizer to increase the denaturation temperature up to $0.96^{\circ} \mathrm{C}$. Penkova et al. [7] have investigated the stabilizing effect of glycerol on the thermal denaturation of several type I collagens in urea ranging from 20 to $50{ }^{\circ} \mathrm{C}$ using a UV-spectroscopy. It was found that the denaturation temperature $\left(T_{\mathrm{d}}\right)$ increased proportionally to the glycerol concentration, indicating that the collagen was stabilized in the presence of glycerol. It was further investigated that the combined effect of glycerol and urea with concentration ranged from 1 to $3 \mathrm{M}$; the results clearly showed that the thermal denaturation induced by urea was improved by the addition of glycerol. Thermal stability of collagen cross-linked with different organic solutions has been studied by C.A. Miles et al., using differential scanning calorimetry (DSC) [8]. It was reported that the improvement in the thermal stability related to the level of fiber dehydration is brought about by the cross-link drawing of collagen molecules. The degree of hydration in collagen could lead to the thermal transition between helix and coil structures [9]. G. Na [10] has studied the interaction of calfskin collagen with glycerol and suggested that the surface interaction of native collagen with glycerol is more favorable than that with water from the three-component solution thermodynamics. Glycerol can be considered as a "thermodynamic inhibitor" against the self-association of fiber collagen since glycerol weakens the fibril assembly reaction through a weak mutual attraction with the protein [10]. $\mathrm{G}$. $\mathrm{Na}$ [10] further proposed that the driving force responsible for the stabilization of the triple-helical structure mainly depends on the interaction of native collagen with glycerol, while it was claimed that 
the denatured collagen unfavorably interacted with glycerol according to the unchanged chemical potential. M. Schoulders et al. [11] also investigated the stability of triple helices and polypeptide chains in collagen, and pointed out that the stabilization is preferentially dependent on the hydrogen bonding, which existed between N-H Gly and neighboring $\mathrm{O}=\mathrm{C}$ strands $[3,10]$.

Recently, an enhanced angular displacement system has been developed using a heterodyne light source and a common-path optical setup for small optical rotation angle detection of glucose [12] and other optically active medium [13]. This is a noninvasive and real-time approach, in which the heterodyne light source consists of two component beams with different frequencies, and the polarization of each component beam is orthogonal to the other. For instance, a beam parallel to the plane of incidence is the TM mode, and the other is the TE mode. Additionally, the heterodyne light source has the electric field represented by the Jones matrices, and is separated into two paths, the measurement path and the reference path, by a beam splitter [13]. However, these true phase measurements are not sensitive enough to detect a small optical rotation angle [14-16]. In this regard, Wu et al., further modified the measurement with a high optical resolution of $1 \times 10^{-40}$ to monitor the blood glucose using an angular displacement-enhanced heterodyne polarimeter [13].

In this paper, we made an attempt to employ an angular displacement-enhanced heterodyne polarimeter to precisely monitor the effect of TFE on the thermal denaturation properties of collagen in rat tails. The TFE aqueous solution with wide range of concentration from $5 \%$ to $80 \%(v / v)$ was used to investigate the interaction of TFE and triple helical structures. Moreover, the inhibiting effect of glycerol on the thermal degradation of TFE-mixed collagen is investigated. The inhibiting mechanism of glycerol with three hydroxyl groups for the stabilization of the triple helix is discussed.

\section{Experimental Section}

\subsection{Experimental Setup}

For the optical measurement, a He-Ne laser with a wavelength of $632.8 \mathrm{~nm}$ was employed as the light source. When the light was passing through the first half wave-plate (HWP1) (NTHU, Hsinchu, Taiwan), the polarization state was adjusted parallel to the incident plane, then divided by a beam splitter (BS1) and fed into a pair of acousto-opto-modulators (AOM1 and AOM2) (NTHU, Hsinchu, Taiwan). A beam combiner (PBS) (NTHU, Hsinchu, Taiwan) was used to combine the output beams from each AOM, and then the heterodyne light source could be obtained. The heterodyne light source consisted of two component beams with frequencies $\omega_{1}$ and $\omega_{2}$, and the polarization of each beam was orthogonal to the other. The one perpendicular to the incidence plane is called the TE mode, while the other is in TM mode. The prototype of an angular displacement-enhanced heterodyne polarimeter has been built and the theoretical analysis has been discussed in [13], in which two angles represent the optical rotation and the phase difference. The relationship between the phase difference $\varphi$ and the intensity of the beam of the laser onto the HWP1 can be expressed as following [13]:

$$
I_{m}=k_{a 1}^{2}+k_{a 2}^{2}+2 k_{a 1} k_{a 2} \cos \left[\left(\omega_{1}-\omega_{2}\right) t+\varphi\right]
$$


where

$$
\begin{aligned}
& k_{a 1}=\left(1-\cos ^{2} \frac{\delta}{2} \sin 2 \beta+\sin ^{2} \frac{\delta}{2} \sin (4 \theta+2 \beta)^{\frac{1}{2}}\right. \\
& k_{a 2}=\left(1+\cos ^{2} \frac{\delta}{2} \sin 2 \beta-\sin ^{2} \frac{\delta}{2} \sin (4 \theta+2 \beta)^{\frac{1}{2}}\right.
\end{aligned}
$$

and phase difference $\varphi$ can be further obtained from

$$
\varphi=\tan ^{-1}\left[\frac{\sin \delta \cos 2 \theta}{\cos ^{2} \frac{\delta}{2} \cos 2 \beta-\sin ^{2} \frac{\delta}{2} \cos (4 \theta+2 \beta)}\right]
$$

The double-distilled water $\left(\mathrm{ddH}_{2} \mathrm{O}\right)$ was injected into the sample tube and used as control. Due to the nature of optical inactivity, the change of the angular displacement is zero (i.e., $\beta=0$ ). The phase difference $\varphi$ can be simplified as the following [13]:

$$
\varphi_{\beta=0}=\tan ^{-1}\left[\frac{\sin \delta \cos 2 \theta}{\cos ^{2} \frac{\delta}{2}-\sin ^{2} \frac{\delta}{2} \cos (4 \theta)}\right]
$$

For all optical measurement, the phase retardation $\delta$ of $178.5^{\circ}$ and azimuth $\theta=23.2^{\circ}$ was set to maintain the larger amplification with great signal-to-noise (SNR). Equation (4) was employed to calculate $\varphi$, between the reference signal and measurement signal, and with adjustment, $\beta$ varied from $0^{\circ}$ to $0.01^{\circ}$ by increasing $0.001^{\circ}$ for each measurement. The amplification factor defined by the ratio of $\varphi$ over $\beta$ is about 42.1 . The thermoelectric cooler (TEC) (NTHU, Hsinchu, Taiwan) was used to heat the sample tube to a temperature from $25{ }^{\circ} \mathrm{C}$ to $55^{\circ} \mathrm{C}$. The temperature deviation during the measurements was less than $\pm 0.3^{\circ} \mathrm{C}$. A Soleil-Babinet compensator (SBC) (NTHU, Hsinchu, Taiwan) was employed to enhance the optical displacement. This SBC was controlled to rotate $0.05^{\circ}$ azimuth degrees per second (i.e., rotate $180^{\circ}$ within $60 \mathrm{~min}$ ), and the sampling frequency is $0.5 \mathrm{~Hz}$. Finally, the phase difference $\varphi$ as a function of phase retardation $\delta$, azimuth $\theta$ of the $\delta$-WP, and angular displacement $\beta$ can be simply read by the lock-in amplifier (LIA). Figure 1 shows a schematic drawing of the applied setup.

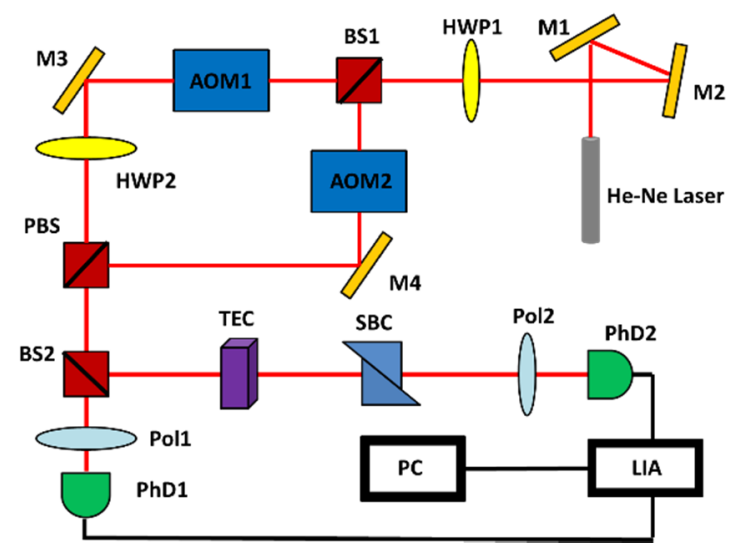

(a)

Figure 1. Cont. 


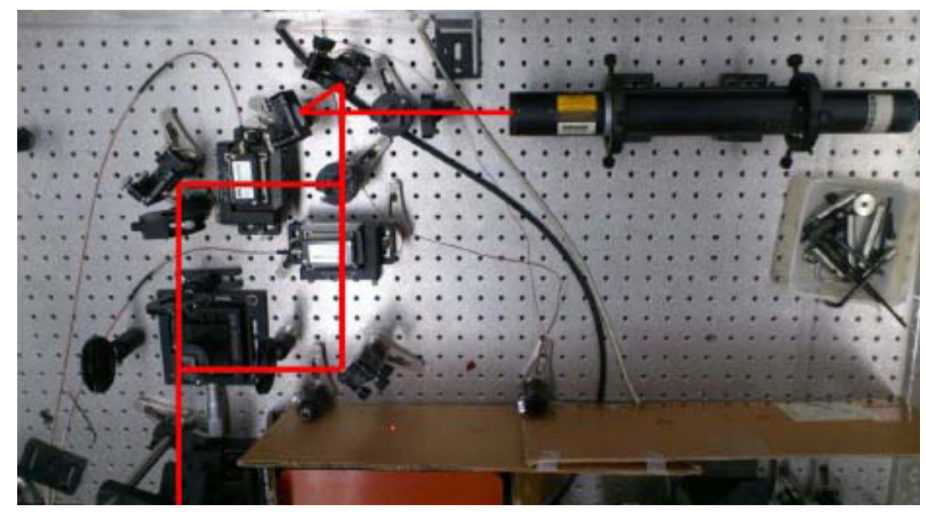

(b)

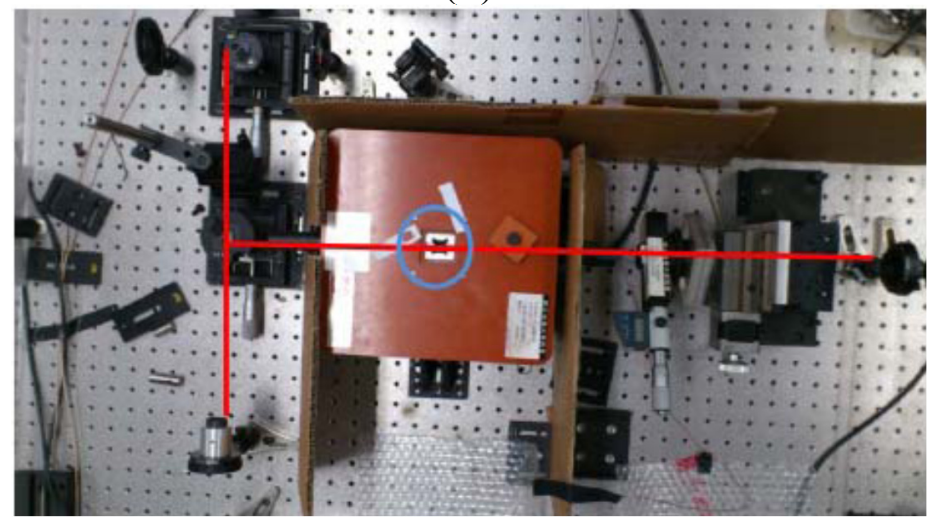

(c)

Figure 1. (a) Schematic diagram of the optical displacement-enhanced heterodyne polarimeter and sampling system; (b,c) show photos of real experimental apparatus and setup. $\mathrm{M}$ : mirror, BS: beamsplitter, PBS: polarizing cube beamsplitter, $\mathrm{PhD}$ : photodetector, SBC: Soleil-Babinet compensator, HWP: half-wave plate, AOM: acousto-optic modulator, Pol: polarizer, TEC: thermoelectric cooler, LIA: lock-in-amplifier, PC: personal computer.

\subsection{Sample Preparation and Measurement of Thermal Denaturation}

The collagen samples purchased from BD Bioscience Co. (Franklin Lakes, NJ, USA) with the product number 354236 are type I collagen extracted from rat tails. The initial concentration was $3.41 \mathrm{mg} / \mathrm{mL}$, which was then diluted to $0.341 \mathrm{mg} / \mathrm{mL}$ using $0.02 \mathrm{M}$ acetic acid ( $\mathrm{pH}$ value $=3.3$ ) as a solvent. Trifluoroethanol (TFE) with 99.9\% purity was purchased from Alfa Aesar Co. (Lancashire, United Kingdom) (product number: 10175784); glycerol (99.9\% in purity) is a product of J. T. Baker Co. (Center Valley, PA, USA) with product number of 2136-01. All reagents were of analytical grade.

For the measurement of the thermal denaturation, the collagen solution with a concentration of $0.341 \mathrm{mg} / \mathrm{mL}$ was mixed with $0.157,0.333,0.529,0.75,2$, and $12 \mathrm{~mL}$ TFE to obtain $5 \%, 10 \%, 15 \%$, $20 \%, 40 \%$, and $80 \%(v / v)$ TFE-mixed collagen solution, respectively. The samples were maintained at $25{ }^{\circ} \mathrm{C}$ for $5 \mathrm{~min}$ and then heated up to $55^{\circ} \mathrm{C}$ with a heating rate of $1{ }^{\circ} \mathrm{C} / \mathrm{min}$ using a TEC. $1 \mathrm{M}$ and $2 \mathrm{M}$ glycerol were used to mix with $10 \%$ and $20 \%(v / v)$ TFE-mixed collagen to investigate the inhibiting effect of the amount of glycerol molecules on the collagen degraded by low and high TFE concentration, respectively. The phase change $\varphi$ was recorded with increasing time and under elevated temperatures as 
well. The denaturation temperature $\left(T_{\mathrm{d}}\right)$ was then determined from a transition point, where a significant temperature drop should be observed from the phase change $v s$. temperature plot.

\section{Results and Discussion}

\subsection{Effect of TFE on the Thermal Denaturation of Collagen}

Figure 2 shows the phase difference $\varphi$ as a function of temperature. The $T_{\mathrm{d}}$ of the native collagen (as a control) is $40.2{ }^{\circ} \mathrm{C}$ from the onset of the significant drop in the phase difference. Figure 3 shows the effect of TFE concentration on the phase difference during the heating process. It can be seen that the onset of the significant drop in the phase difference is shifted to a lower temperature, indicating that the $T_{\mathrm{d}}$ of the TFE-mixed collagen is decreased from $37.3{ }^{\circ} \mathrm{C}$ to $28.4{ }^{\circ} \mathrm{C}$. In this stage, the addition of TFE increases the heterogeneity of collagen in the aqueous buffer solutions due to the formation of unordered peptide carbonyl groups $(-\mathrm{C}=\mathrm{O})[4,17]$. As the TFE concentration is higher than $40 \%(v / v)$, the significant drop in the phase difference diminishes and reveals that the collagen has been completely destructed. It has been suggested that the variation in the optical properties can be used to explore the thermal degradation of TFE-mixed collagen and the resulting structural destabilization [4]. Thus, we recorded the initial optical rotation as a function of TFE concentration from the displacement-enhanced heterodyne measurement, as shown in Figure 4a. It can be seen that initial optical rotation is reduced by $0.1^{\circ}$ as TFE concentration increased to $15 \%(v / v)$, while this value is significantly reduced to less than $0.4^{\circ}$. It has been suggested that TFE introduces the $\alpha$-helical structure in the aqueous TFE solution, and then causes the disruption of the inter-strand hydrogen boding (i.e., the bifurcated hydrogen bonding for $\mathrm{C}=\mathrm{O}$ chains between the $\mathrm{N}-\mathrm{H}$ and $\mathrm{O}-\mathrm{H}$ group of TFE). As the TFE concentration further increases $(40 \% \sim 80 \%, v / v)$, the initial optical rotation of the TFE-mixed collagen remains almost unchanged, indicating that the inter-strand hydrogen bonding between the $\mathrm{N}-\mathrm{H}$ Gly and neighboring $\mathrm{C}=\mathrm{O}$ Xaa groups has been destructed and the fabrils in the collagen have been completely inhibited [4,18-20]. In this way, the triple helices have become destabilized by transforming the helical structure into an unordered structure, as proposed by Shanmugam [4] and Rajan [18]. The dependence of the denaturation temperature on the TFE concentration is illustrated in Figure $4 \mathrm{~b}$, revealing that $T_{\mathrm{d}}$ of the TFE-mixed collagen decreased proportionally to the TFE concentration in the solution.

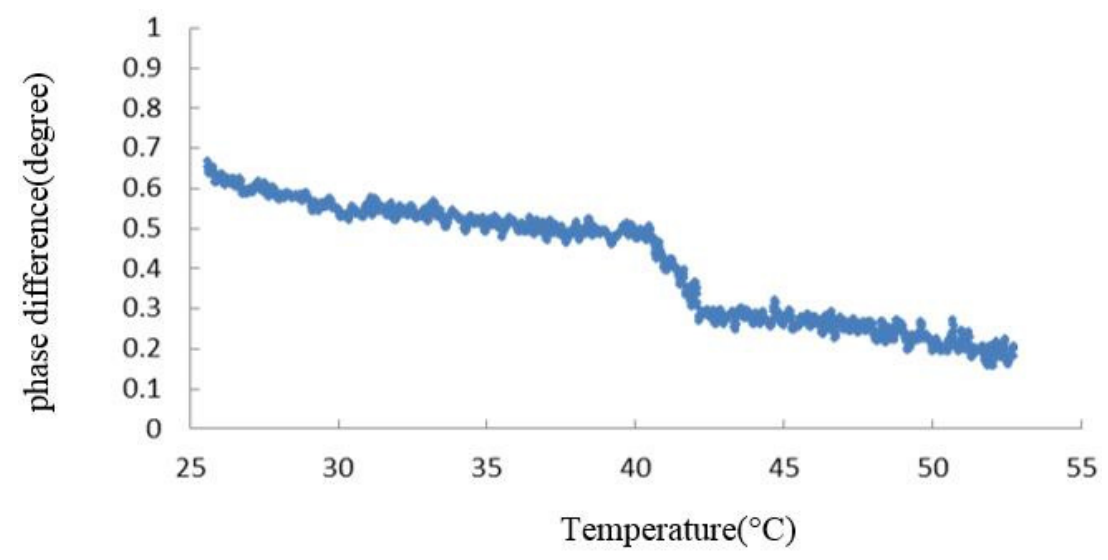

Figure 2. The phase difference of collagen as a function of temperature. (Collagen concentration: $0.341 \mathrm{mg} / \mathrm{mL}$ ). 

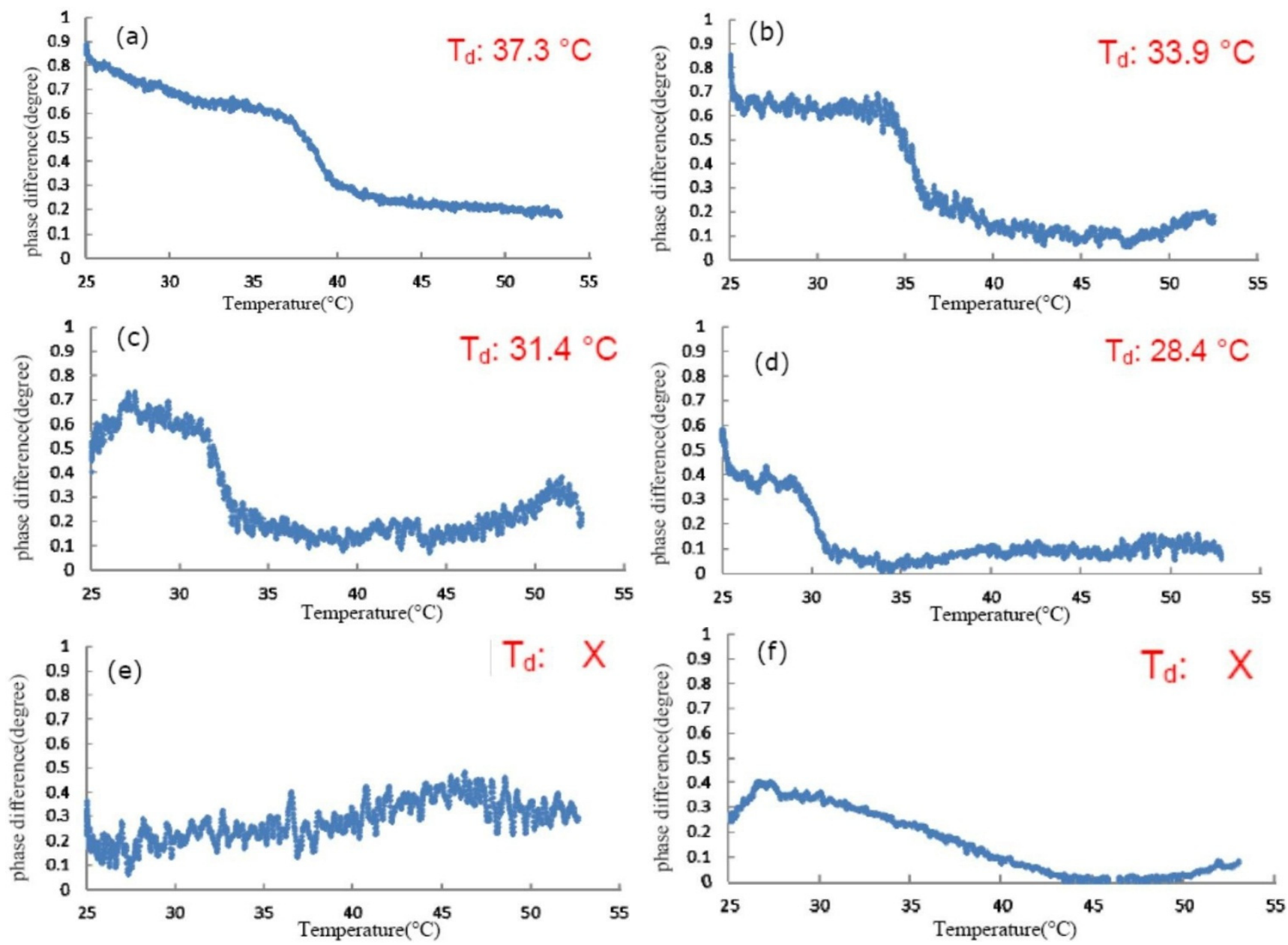

Figure 3. Variation of phase difference with heating temperature under different Trifluoroethanol (TFE) concentration $(v / v)$ : (a) $5 \%(=0.157 \mathrm{~mL})$; (b) $10 \%(=0.333 \mathrm{~mL})$; (c) $15 \%(=0.529 \mathrm{~mL})$; (d) $20 \%(=0.75 \mathrm{~mL})$; (e) $40 \%(=2 \mathrm{~mL})$ and (f) $80 \%(=12 \mathrm{~mL})$. (Collagen concentration: $0.341 \mathrm{mg} / \mathrm{mL}$ ) The inset $T_{\mathrm{d}}$ represents the denaturing temperature.
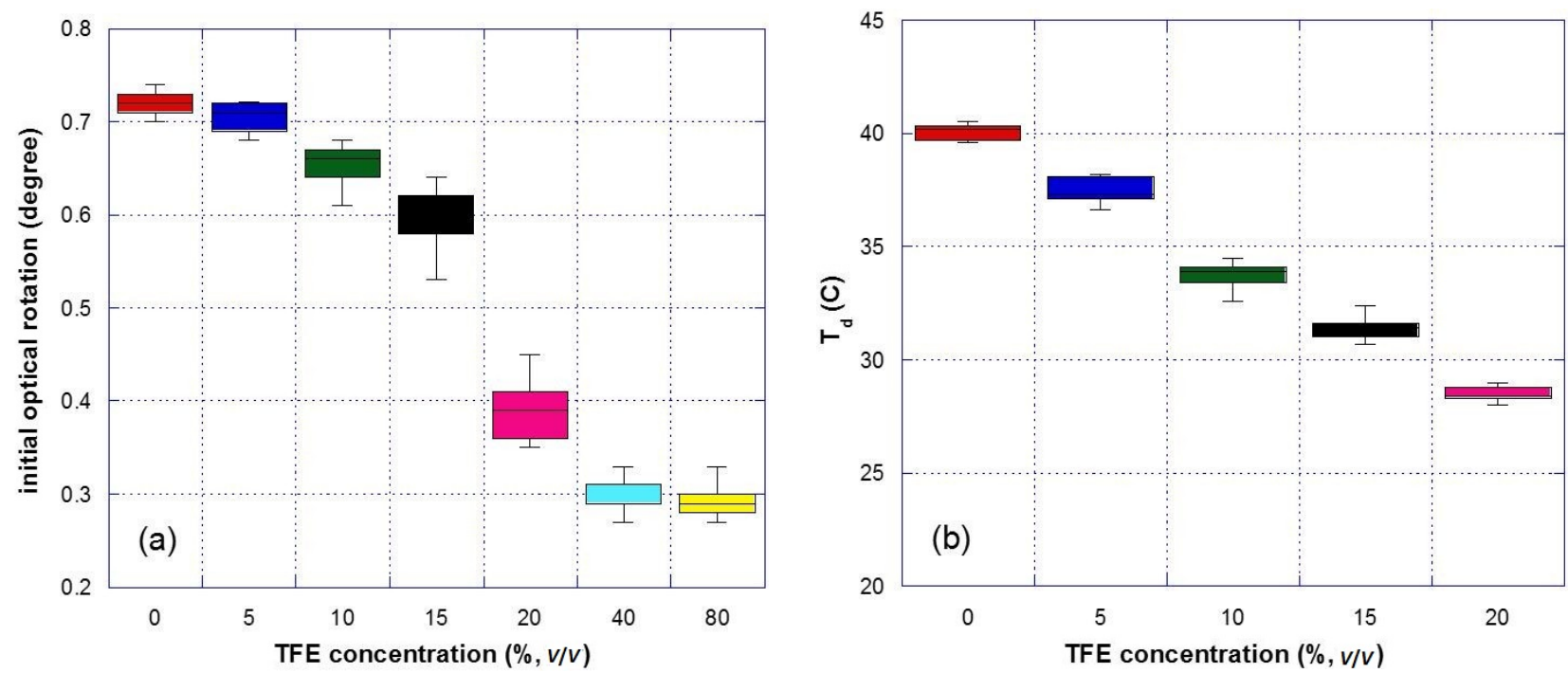

Figure 4. (a) Initial optical rotation and (b) denaturation temperature $\left(T_{\mathrm{d}}\right)$ as a function of TFE concentration $(\%, v / v)$. (Collagen concentration: $0.341 \mathrm{mg} / \mathrm{mL})$. 


\subsection{Effect of Glycerol on the Thermal Denaturation of Collagen and TFE-Mixed Collagen}

We first investigate the effect of glycerol on the thermal denaturation of collagen. As seen in Figure 5, the $T_{\mathrm{d}}$ is increased by increasing glycerol concentration. As for the $3 \mathrm{M}$ glycerol, the $T_{\mathrm{d}}$ is increased to $42.4^{\circ} \mathrm{C}$ and the stabilization of collagen is improved. Figure 6 shows the inhibiting effect of glycerol on the collagen degraded by $10 \%(v / v)$ TFE. It can be seen that the initial optical rotation is restored and the $T_{\mathrm{d}}$ value is increased to $36.4{ }^{\circ} \mathrm{C}$ as compared to the value of TFE-mixed collagen $\left(T_{\mathrm{d}}=33.9^{\circ} \mathrm{C}\right)$. This result provides evidence that glycerol has effective inhibiting abilities on the thermal denaturation of collagen degraded by TFE, even under low glycerol concentration. We further examine the inhibiting effect of glycerol on the high-concentration TFE $(20 \%, v / v)$ mixed collagen, wherein the helical structures have been almost destructed. As shown in Figure 7, $1 \mathrm{M}$ and $2 \mathrm{M}$ glycerol can increase the $T_{\mathrm{d}}$ to $31.3{ }^{\circ} \mathrm{C}$ and $32.8^{\circ} \mathrm{C}$, respectively. Figure 8 shows the degradation of $T_{\mathrm{d}}$ in the presence of TFE and the inhibiting effect of glycerol under different concentrations. The result indicates that glycerol increases $T_{\mathrm{d}}$ by $2.2^{\circ} \mathrm{C}$ in the absence of TFE, and further increases the $T_{\mathrm{d}}$ of TFE-mixed collagen at high concentration by $4.2{ }^{\circ} \mathrm{C}$. This improvement is in agreement with the fact that glycerol plays a role of stabilizer in the calfskin collagen solution and increases its $T_{\mathrm{d}}$ by $0.96{ }^{\circ} \mathrm{C}$ [6]. It has been suggested that the inhibiting effect of glycerol on the thermal denaturation is achieved by the incorporation of its hydroxyl groups into the water-collagen molecular structure [7]. In other words, glycerol binds to the surface of collagen molecules through the formation of hydrogen bonding, which competes with the water molecules as a replacement for water-mediated hydrogen bonds. Subsequently, the extensive cross-linking structure inhibits collagen dissociation by glycerol [5]. Na obtained a negative chemical potential change of collagen binding with glycerol molecules using the Wyman linked function, and reported that the triple helical structure can be preserved due to the fact that glycerol interaction with the surface of native collagen is more energetically favorable than the interaction between water and collagen [10]. With increasing glycerol concentration, a large amount of hydroxyl groups containing additional and stabilizing hydrogen bonds is generated and leads to greater ability in the inhabitation of thermal denaturation. Similar results can be observed for the stabilizing effect of glycerol on the calfskin collagen degraded by urea [7], where about $1{ }^{\circ} \mathrm{C}$ increase of the denaturation temperature per $1 \mathrm{M}$ glycerol was reported.
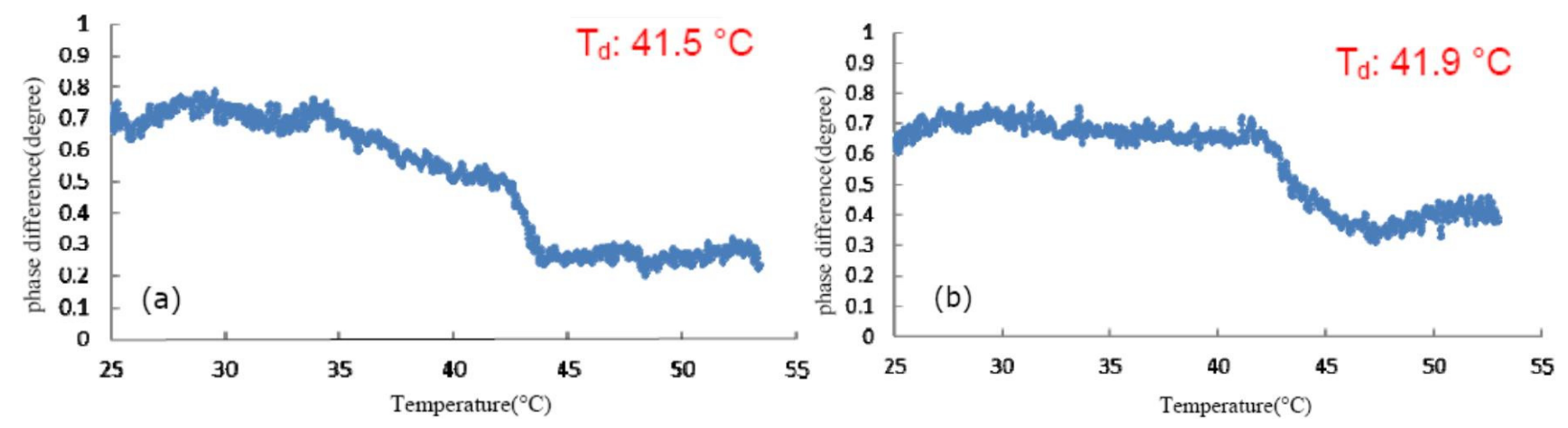

Figure 5. Cont. 


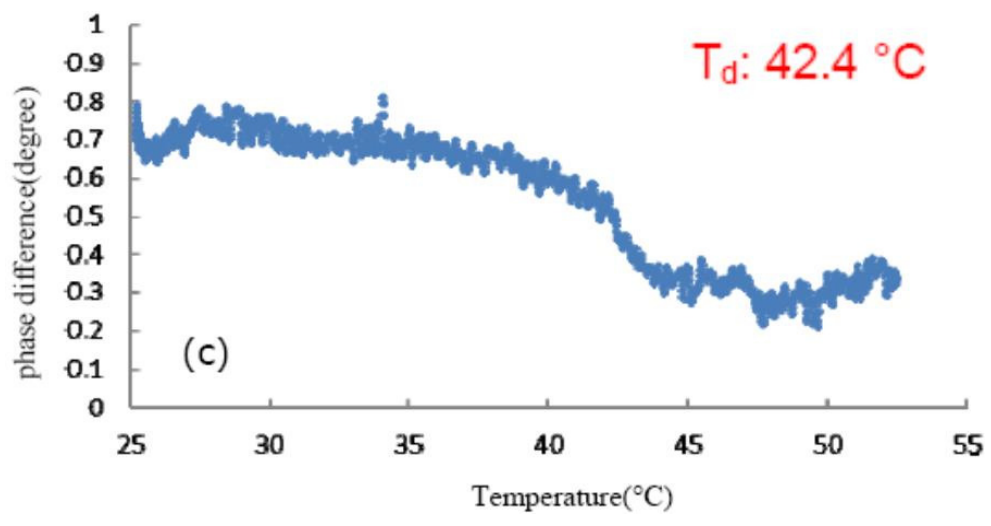

Figure 5. Effect of glycerol on phase difference of collagen as a function of temperature under (a) $1 \mathrm{M}$; (b) $2 \mathrm{M}$; (c) $3 \mathrm{M}$ glycerol concentration (Collagen concentration: $0.341 \mathrm{mg} / \mathrm{mL}$ ).
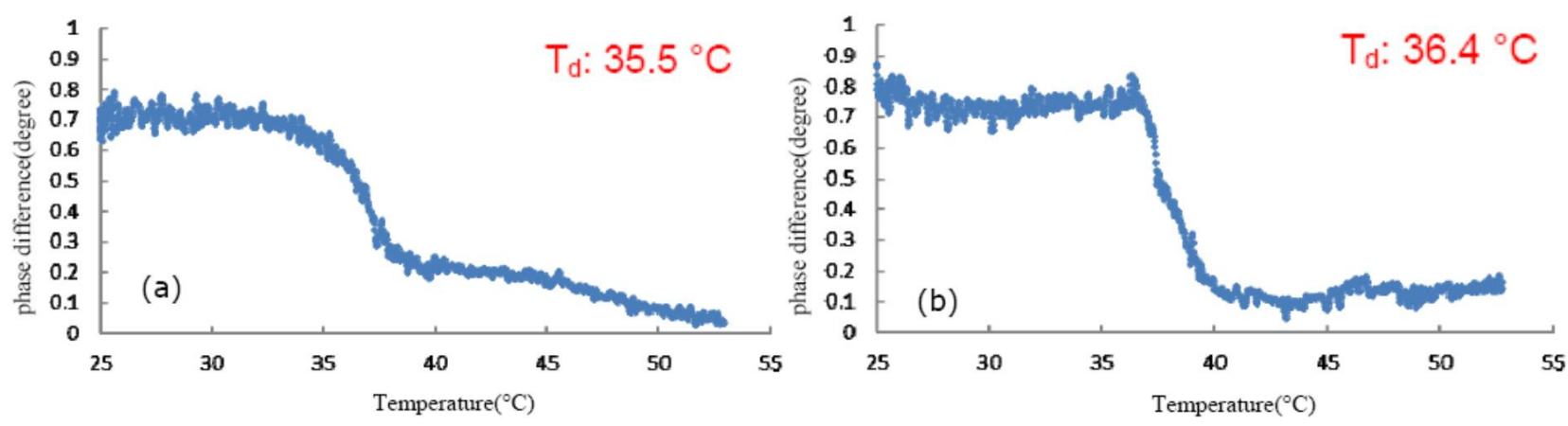

Figure 6. Effect of glycerol on phase difference of TFE $(10 \%, v / v)$-mixed collagen as a function of temperature under different glycerol concentration: (a) $1 \mathrm{M}$; (b) $2 \mathrm{M}$ (Collagen concentration: $0.341 \mathrm{mg} / \mathrm{mL}$ ). The inset $T_{\mathrm{d}}$ represents the denaturing temperature.
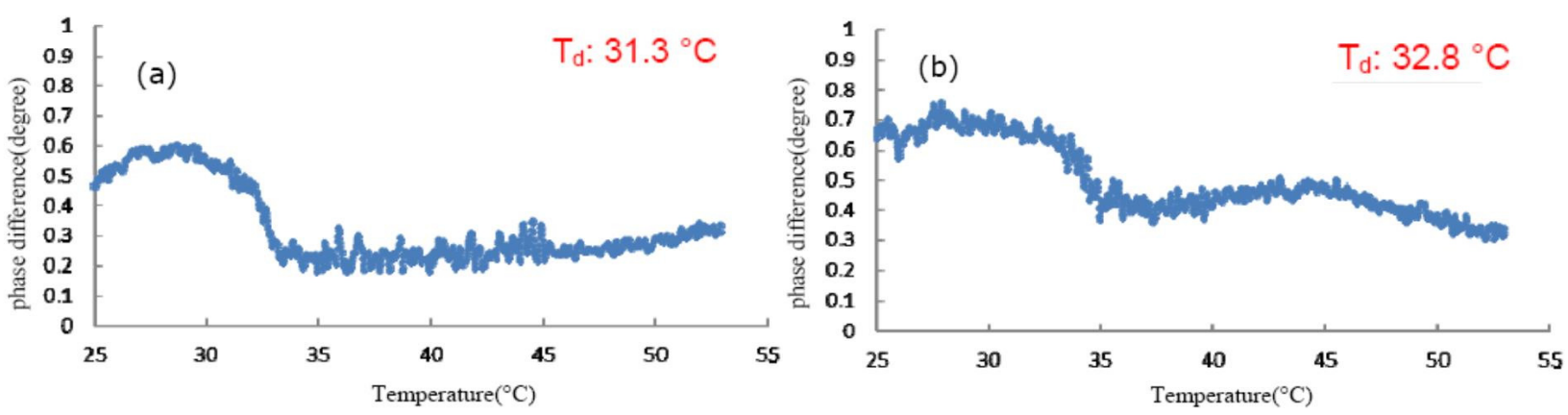

Figure 7. Effect of glycerol on phase difference of TFE $(20 \%, v / v)$-mixed collagen as a function of temperature under (a) $1 \mathrm{M}$; (b) $2 \mathrm{M}$. glycerol concentration (Collagen concentration: $0.341 \mathrm{mg} / \mathrm{mL}$ ). The inset $T_{\mathrm{d}}$ represents the denaturing temperature. 


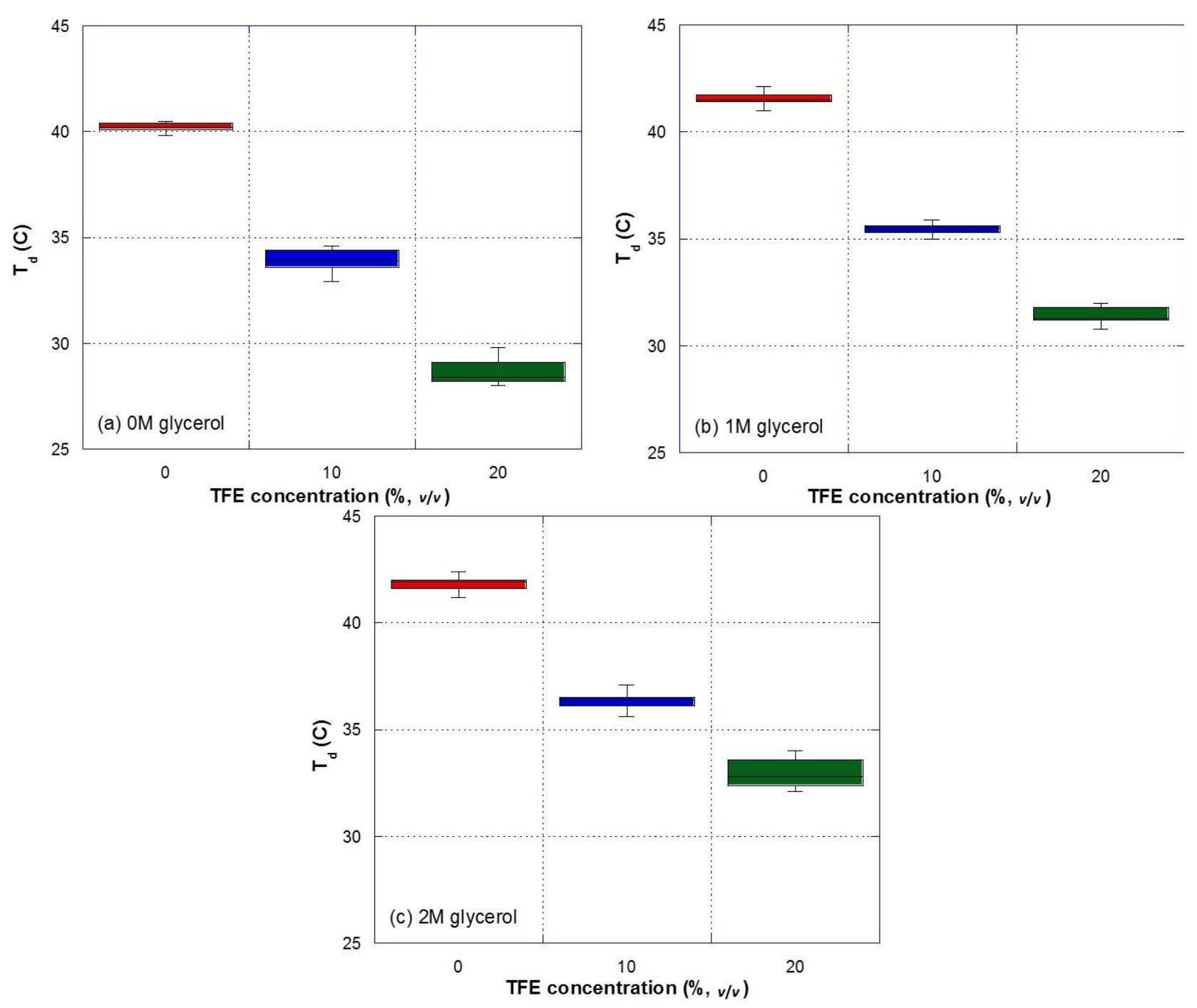

Figure 8. Stabilizing effect of glycerol in the presence of TFE on rat tail collagen. (a) $0 \mathrm{M}$ glycerol; (b) 1 M glycerol; (c) 2 M glycerol.

\section{Conclusions}

The effect of TFE on the optical properties and thermal denaturation of rat tail collagen have been investigated using an angular displacement-enhanced heterodyne polarimeter. The present results indicate that TFE affects the optical rotation and decreases the denaturation temperature due to the disruption of the inter-strand hydrogen bonding. As a result, the triple helices are transformed to an unordered structure in collagen, as evidenced by the decrease in the optical rotation. It is clearly observed that the stability of triple helices is significantly reduced at a TFE concentration higher than $15 \%(v / v)$. Furthermore, we demonstrated the inhibiting effect of glycerol on the thermal denaturation of the TFE-mixed collagen. It has been shown that the $T_{\mathrm{d}}$ is significantly increased with increasing glycerol concentration, indicating the TFE-mixed collagen has been stabilized through the extensive covalent cross-links in the presence of glycerol. The relationship between TFE and glycerol concentration on the optical properties is presented; the $T_{\mathrm{d}}$ can be restored to $32.8^{\circ} \mathrm{C}$ by glycerol at $2 \mathrm{M}$ and even under high TFE concentration $(20 \%, v / v)$. 


\section{Acknowledgments}

This research was funded by the National Science Council of Taiwan, ROC, under contract No. NSC 102-2221-E-007-110.

\section{Author Contributions}

Chien-Ming $\mathrm{Wu}$ and Horn-Haw Chen performed the experimental setup and model construction; Kai-Han Tseng performed the experiment of TFE effect and data collection; Hung-Wei Chen integrated the optical measurement, performed model construction, analyzed experimental data and prepared manuscript/figures preparation.

\section{Conflicts of Interest}

The authors declare no conflict of interest.

\section{References}

1. Zhong, S.; Teo, W.E.; Zhu, X.; Beuerman, R.; Ramakrishna, S.; Yung, L.Y.L. Formation of collagen-glycosaminoglycan blended nanofibrous scaffolds and their biological properties. Biomacromolecules 2005, 6, 2998-3004.

2. Zhong, S.P.; Teo, W.E.; Zhu, X.; Beuerman, R.; Ramakrishna, S.; Yung, L.Y.L. Development of a novel collagen-GAG nanobibrous scaffold via electrospinning. Mater. Sci. Eng. C 2007, 27, 262-266.

3. Zeugolis, D.I.; Khew, S.T.; Yew, E.S.; Ekaputra, A.K.; Tong, Y.W.; Yung, L.Y.; Hutmacher, D.W.; Sheppard, C.; Raghunath, M. Electro-spinning of pure collagen nano-fibres-Just an expansive way to make gelatin? Biomaterials 2008, 29, 2293-2305.

4. Shanmugam, G.; Reddy, S.M.M.; Natarajan, V.; Madhan, B. 2,2,2-Trifluoroethanol disrupts the triple helical structure and self-association of type I collagen. Int. J. Biol. Macromol. 2013, 54, 155-159.

5. Yeh, A.T.; Choi, B.; Nelson, J.S.; Tromberg, B.J. Reversible dissociation of collagen in tissues. J. Invest. Dermatol. 2003, 121, 1332-1335.

6. Cooper, D.R.; Russell, A.E. Hart, G.J. The effects of glycols on the renaturation of soluble collagen. Biochem. J. 1971, 125, 1069-1074.

7. Penkova, R.; Goshev, I.; Gorinstein, S.; Nedkov, P. Stabilizing effect of glycerol on collagen type I isolated from different species. Food Chem. 1999, 66, 483-487.

8. Miles, C.A.; Avery, N.C.; Rodin, V.V.; Bailey, A.J. The increase in denaturation temperature following cross-linking of collagen is caused by dehydration of the fibres. J. Mol. Biol. 2005, 346, 551-556.

9. Sionkowska, A. Thermal denaturation of UV-irradiated wet rat tail tendon collagen. Int. J. Biol. Macromol. 2005, 35, 145-149.

10. Na, G.C. Interaction of calf skin collagen with glycerol: linked function analysis. Biochemistry 1986, 25, 967-973. 
11. Shoulders, M.D.; Rains, R.T. Collagen structure and stability. Annu. Rev. Biochem. 2009, 78, 929-958.

12. Klonoff, D.C. Noninvasive blood glucose monitoring. Diabetes Care 1997, 20, 433-437.

13. Wu, C.M.; Tsai, Y.C. Angular displacement-enhanced heterodyne polarimeter for the measurement of optically active media. Sens. Actuators B 2006, 120, 324-328.

14. Cote, G.L.; Fox, M.D.; Northrop, R.B. Noninvasive optical polarimetric glucose sensing using a true phase measurement technique. IEEE Trans. Biomed. Eng. 1992, 39, 752-756.

15. Chou, C.; Huang, Y.C.; Chang, M. Precise optical activity measurement of quartz plate by using a true phase-sensitive technique. Appl. Opt. 1997, 36, 3604-3609.

16. Feng, C.M.; Huang, Y.C.; Chang, J.G.; Chang, M.; Chou, C. A true sensitive optical heterodyne polarimeter for glucose concentration measurement. Opt. Commun. 1997, 141, 314-321.

17. Williams, B.R.; Gelman, R.A.; Poppke, D.C.; Piez, K.A. Collagen fibril formation. Optimal in vitro conditions and preliminary kinetic results. J. Biol. Chem. 1978, 253, 6578-6585.

18. Rajan, R.; Balaram, P. A model for the interaction of trifluoroethanol with peptides and proteins. Int. J. Peptide Protein Res. 1996, 48, 328-336.

19. Bodkin, M.J.; Goodfellow, J.M. Hydrophobic salvation in aqueous trifluoroethanol solution. Biopolymers 1996, 39, 43-50.

20. Kotch, F.W.; Raines, R.T. Self-assembly of synthetic collagen triple helices. Proc. Natl. Acad. Sci. USA 2006, 103, 3028-3033.

(C) 2015 by the authors; licensee MDPI, Basel, Switzerland. This article is an open access article distributed under the terms and conditions of the Creative Commons Attribution license (http://creativecommons.org/licenses/by/4.0/). 